\title{
MÁS ALLÁ DE LA ACCIÓN CULTURAL DEL ESTADO. APUNTES PARA UNA EVOLUCIÓN DE LAS POLÍTICAS CULTURALES EN CHILE*
}

\author{
BEYOND THE STATE'S CUltural ACTION. NOTES \\ FOR AN EVOLUTION OF CULTURAL POLICIES IN CHILE
}

\section{CRISTIAN ANTOINE**}

\section{RESUMEN}

Pese a que el Estado chileno viene desarrollando una nutrida actividad en el campo cultural desde los orígenes de la República, la creación en 2003 del Consejo Nacional de la Cultura y las Artes no ha supuesto necesariamente un desarrollo formal de políticas públicas en cultura ( $p$ ublic policies in culture), especialmente porque estas decisiones legislativas, financieras y administrativas que el Estado realiza en el campo cultural no son sujetas a mecanismos conocidos de evaluación ni accountability. Cuando mucho lo que han hecho algunos gobiernos es presentar episódicos balances de la acción cultural que realizan con cargo al erario público. El ensayo aporta una propuesta de periodización de la evolución de la acción pública del Estado chileno en cultura, explorando algunas razones por las que no se ha avanzado en una conceptualización más moderna de las políticas culturales.

Palabras clave: Políticas culturales, evaluación, políticas públicas, acción cultural del Estado.

\section{ABSTRACT}

Even though the Chilean State has developed sustained activity in culture since the founding of the Republic, these advances have not necessarily crystallized in a formal development of public policies in culture, especially when the legislative, financial and

* Este trabajo está asociado al Proyecto Fondecyt Regular 2008 No 1.085.049 “Análisis de las Políticas Culturales en Chile: Consumidores y Nuevas Audiencias”, Escuela de Periodismo, Universidad de Santiago de Chile.

${ }^{* *}$ Historiador, periodista, investigador y docente. Escuela de Periodismo, Facultad de Comunicaciones, Universidad del Pacífico. Santiago, Chile. Correo: cantoine@upacifico.cl 
administrative decisions the State has made are not subject to known mechanisms of evaluation or accountability. This is true even when taking into account the creation in 2003 of the Consejo Nacional de la Cultura y las Artes. At the most, what some governments have presented are occasional public balance sheets of cultural actions that are carried out using public funding. This essay proposes the division into separate periods of the evolution of public action on the part of the Chilean State in culture, exploring some reasons why there has been little or no advancement towards a more modern conceptualization of cultural policies.

Keywords: Cultural policies, assessment, public policies, State's cultural actions.

Recibido: 18.03.11. Aceptado: 04.08.11.

\section{INTRODUCCIÓN}

$\mathrm{E}$ N LOS ÚlTIMOS CINCUENTA AÑOS el acceso a la cultura y las artes en las sociedades actuales ha pasado de un privilegio de los grupos dominantes a ser considerada como un derecho fundamental, individual y colectivo y un sector clave para el desarrollo de la comunidad. Las políticas culturales tienen, en este contexto, un importante rol que cumplir, en cuanto son la manera concreta como los distintos países se organizan para satisfacer los derechos culturales de los ciudadanos. ¿Pero, de qué hablamos cuando hablamos de políticas culturales? ¿Son acaso las políticas culturales genuinas representaciones institucionales de lo que se ha dado en llamar "políticas públicas", en este caso en cultura?

Dicho de otra forma, ¿son acaso todas las "políticas" que un gobierno desarrolla homologables como "políticas públicas"?

Es frecuente que desde una perspectiva generalizadora se entienda a las "políticas públicas" como "aquello que los gobiernos hacen". Ese es el sentido más obvio que la expresión "política" tiene en nuestro idioma (del lat. politǐcus, y este del gr. $\pi \mathrm{o} \lambda \iota \tau \iota \kappa \varsigma)$, de donde la política es aquello perteneciente o relativo a la doctrina política; a la actividad política o que interviene en las cosas del gobierno y negocios del Estado, los Estados.

No obstante, en la tradición anglosajona de los estudios sobre políticas públicas (Atlas, 2002; Bennett, 2009), el término "política" tiene dos significados: primero, el de politics, asumido como conjunto de procedimientos de lucha por alcanzar el poder (lo que alude a los aspectos más negativos de la confrontación ideológica) y, segundo, como policy (policies en plural), con el sentido de acción del gobierno y sus distintos programas de intervención en "lo público". 
Nuestro idioma no hace diferencia entre estos dos sentidos. Allí donde los anglosajones ven politics y policy, nosotros vemos solo "política". Y claramente no da lo mismo.

Así, cuando pretendemos estudiar la política en el sentido de politics, nuestros objetos de estudio son las fuerzas políticas, los partidos, las elecciones, las instituciones gubernamentales y parlamentarias. Mientras que cuando estudiamos las políticas públicas como "policies" (Bradford et al., 2000; Mark, 1969) estamos más preocupados de observar las decisiones formales que los gobiernos asumen, lo que implica simultáneamente a diversos actores, aquellos que la adoptan y aquellos que resultan afectados por ellas.

Existe cierta consenso en considerar el aspecto institucional que toda policy comporta, pues se trata de la decisión de una autoridad legitima, adoptada dentro de un campo reconocido de decisión y siguiendo procedimientos legalmente establecidos.

Por policy, que proponemos comprender aquí por políticas públicas o por el término de política seguido de un adjetivo (social, agrícola, cultural, etcétera), hacemos referencia a la acción concreta del Estado, al aspecto programático de la acción gubernamental sobre un ámbito específico de la vida social sobre el cual se ha decidido intervenir con criterios de racionalidad y la disposición a dar debida cuenta de sus resultados.

Así, por ejemplo, desde la creación en 2003 del Consejo Nacional de la Cultura y las Artes (CNCA), la administración chilena viene desarrollando una nutrida actividad en el ámbito artístico y cultural -vg., dictando leyes específicas para el sector, creando fondos económicos para su financiamiento y disponiendo medidas administrativas en su concurso- generando, sin duda, "políticas culturales". Pero, habida cuenta de la falta de evaluación y de la aparente incapacidad del sector público de poder generarla, es dable plantearse si éstas corresponden en realidad a "políticas públicas en cultura" (cultural public policies).

En el presente trabajo nos proponemos asumir esta cuestión. Primeramente revisaremos los aspectos doctrinales que permiten considerar a la evaluación y la rendición de cuentas de las políticas públicas como un requisito sine qua non de las mismas. En seguida, reseñaremos aquellos que nos parecen han sido los hitos que configuran la evolución de las políticas culturales chilenas y que nos ayudan a poner en contexto la pertinencia de asumir tal denominación para la acción cultural que realiza el Estado en Chile. 


\section{LAS POLÍTICAS PÚBLICAS Y SU EVALUACIÓN}

No existe una definición de consenso sobre lo que son las políticas públicas. La noción de política pública tiene acercamientos descriptivos y teóricos. Quién, cómo y para qué detenta el poder político colectivamente vinculante, a la base y acaso al margen de la autoridad estatal formalmente constituida, ha sido la pregunta central y también recelosa de la cienciasociología política (Aguilar Villanueva, 1992; Ballart, 2005).

En la opinión de Quade (1989) no hay ninguna diferencia sustantiva entre el análisis de sistemas y el análisis de políticas, con excepción de una mayor complejidad al intervenir consideraciones sociales y políticas.

Contra tal enfoque reaccionó Charles Lindblom (1996) apoyándose en la tesis de la sociedad abierta de Popper, para quien ni las políticas ni las políticas públicas, en sus fines o en sus medios, podían pretender basarse en conocimientos de validez absoluta, como quería hacer creer el autoritarismo de los planificadores centrales. Lindblom afirmó que los gobiernos podían decidir racional y responsablemente frente a los problemas públicos sin tener que comprometerse con los supuestos y requisitos de la racional ilimitada. La clave estaba en la evaluación. De hecho, el mismo Harold Lasswell había sostenido que el proceso de elaboración de políticas y programas es racional y ordenado (Lasswell, 1971; Lasswell \& Aguilar Villanueva, 1992).

Pero los elementos que entran en juego son muy variados, mucho más allá de los gobiernos y de quienes administran el aparato del Estado. Las políticas públicas reflejan procesos decisorios y dinámicos de toma de decisiones racionales por parte del poder. Estos procesos se producen en gran parte por instancias organizativas públicas que involucran a las instituciones del Estado con la mayoría de las cuestiones importantes de nuestra sociedad: la estratificación económica, las relaciones internacionales, la tecnología, el turismo, el patrimonio, la educación y el desarrollo comunitario, sólo por nombrar a las más relevantes.

La racionalidad en la toma de las decisiones para la conformación de las políticas públicas en América Latina se instaló tras la aparición de diversos Gobiernos de corte militar; administraciones que promovieron un enfoque relativamente cerrado y tecnocratizado de diseñar políticas públicas. Racionalidad es asumida aquí como el examen de las relaciones entre las necesidades y/o problemas detectados en el ámbito espacial donde se ejecuta el programa (y para una población objetivo determinada) con los objetivos establecidos para la mejora o solución de dichos problemas. Implica además el análisis de la relevancia y pertinencia de un programa. 
Las políticas públicas tienen que ver entonces con el uso estratégico de los recursos para aligerar los problemas sociales y con el conjunto de las actividades de las instituciones de gobierno actuando directamente o a través de agentes y que van dirigidas a tener una influencia determinada sobre la vida de los ciudadanos (Gelambi, 1999).

Así, generalmente por "políticas públicas" se han entendido los programas que un gobierno, cualquiera que sea, desarrolla en función de un problema o situación determinada (Huerta, 2004). Vega \& Bedregal (2010) han explicado cómo en los últimos años ha crecido el interés entre académicos y formuladores de políticas, por el uso o no uso de la evidencia científica en la elaboración de las mismas. Dicho interés, se ha dicho (Burlot \& Medalla, 2008) se enmarca en el paradigma del diseño de las políticas públicas basadas en la evidencia, dentro del cual se espera que la certeza científica las inspire para que éstas sean las más efectivas y eficaces estrategias a implementar.

Aunque sea difícil definirlas, sí existe bastante consenso en las etapas que incluye su proceso vital. No hay autores que no sostengan que el final del proceso (o el principio del mismo según se prefiera) tanto del diseño como de la implementación de las políticas públicas, ha de incluir siempre y necesariamente la práctica de la evaluación.

Si las políticas públicas descansan entonces en la aplicación de la razón a la acción de los Estados, evaluar los efectos de las intervenciones públicas contribuirá entonces a mejorar la racionalidad instrumental de la implementación de las decisiones de los gobiernos (Rausell Koster, 2004), permitiendo conocer mejor la realidad sobre la que el decidor estatal interviene (comparación y objetivos), e instando a la realización de análisis de la eficacia de la acción gubernamental. En general, las políticas que no pueden concretar objetivos en indicadores más o menos cuantificables, difícilmente pueden ser buenas intervenciones. Su idoneidad dependerá de la interpretación arbitraria de un agente interesado, lo que reduce el calado democrático de las intervenciones públicas y disminuye el papel de la participación social.

Asegurar una mejor gestión y dar cuenta pública (accountability) a los ciudadanos son imperativos indiscutibles en una sociedad democrática donde la legitimidad de las decisiones es cuestionada permanentemente (Ingram, Louis, \& Schroeder, 2004)․․ Además de medir los costes y los be-

${ }^{1}$ Accountability / responsabilidad / rendición de cuentas es un concepto ético que nace en el Institute of Social and Ethical Accountability, que desde 1996 busca promover la rendición de cuentas. Existen ocho tipos de rendición de cuentas: moral, administrativo, político, de gestión, comercialización, jurídico y judicial, circunscripción relación y profesional. 
neficios de las políticas públicas, la evaluación pretende aclarar lo que está en juego y los mecanismos a la obra con el fin de permitir tanto a los cargos electos como a los ciudadanos convertirse en actores informados y responsables. La evaluación y la construcción de indicadores para las políticas públicas pasa a ser entonces uno de los elementos sine qua non de su proceso de diseño e implementación ${ }^{2}$ (Yoshimoto, 2005).

Una política cultural ${ }^{3}$, como cualquier otra política pública, debería consistir en una acción de gobierno basada en el análisis de la realidad (Yúdice \& Miller, 2004) y en un programa que pretende obtener unos objetivos de cambio y mejora (Ochoa Gautier, 2003) contrastables con la realidad a partir de procesos objetivos de evaluación. Si aspira a ser una política pública, la política cultural debiera entonces considerar mecanismos de evaluación y seguimiento.

Caso contrario, las formulaciones contenidas como objetivos a lograr en la formulación de las políticas, no quedarán sino circunscritas al mero ámbito de la expresión de deseos. Es necesario considerar que las metas en políticas públicas debieran atenderse (Solarte Pazos, 2004; Vedung, 1986) como "índices que se pueden lograr o realizar mediante las políticas". Ellas pueden ser formuladas indicando la naturaleza existente de los cambios que se pueden o se aspira a lograr. Si, en efecto, cada programa político comprende una jerarquía de metas y medios deseables, en otro nivel pueden también simultáneamente ser un medio.

\footnotetext{
2 "La evaluación se aplica a objetos de naturaleza distinta (plan, programa, proyecto), a niveles administrativos diferentes (local, regional, nacional, europeo) y a distintos ámbitos (sanidad, educación, medioambiente, seguridad). Puede tener diferentes finalidades, tanto explícitas como implícitas: ayuda a la decisión, informe, gestión, mediación, aprendizaje; lo que provoca una gran diversidad de métodos. Para una misma política, las informaciones que conviene privilegiar y los puntos de vista a tener en cuenta no son los mismos según que la evaluación pretende dar cuenta de los resultados o aclarar el proceso de aplicación. A pesar de que no exista ningún método que permita evaluar todos los planes o programas, es posible definir unos principios de conducta de una operación de evaluación suficientemente general para adaptarse a una amplia gama de situaciones". Cfr U. Urteaga (2009).

${ }^{3}$ Definidas por Ander-Egg (2005: 230) como "El conjunto de operaciones, principios, prácticas y procedimientos de gestión administrativa o presupuestaria que sirven de base a la acción cultural del Estado", las políticas culturales del Estado cumplen un papel en relación a la cultura de una sociedad, aunque se debe concluir también que a su lado hay otros actores como los creadores y el sector privado (Ander-Egg, 2005). Para Garretón, las políticas culturales son el conjunto de actividades e iniciativas dirigidas a satisfacer necesidades culturales, desarrollar el ámbito expresivo-simbólico y generar perspectivas compartidas de la vida social, de una determinada comunidad. La idea de políticas culturales, en general referidas al Estado, es más bien de corta data, aun cuando haya habido políticas culturales de los Estados y de los gobiernos desde que éstos existen (Garreton, 1992).
} 
Lo dable sería esperar, junto a la formulación de las mismas, una relación sobre cuestiones como: ¿Se han logrado las metas del (o los) programa (s)?; ¿cuáles son los costos y efectos colaterales de los programas adoptados?; ¿por qué las metas se han o no logrado?; ¿los programas han contribuido al logro de la meta en una forma eficiente?; ¿ bajo qué situaciones los programas de políticas culturales tienen éxito o fracasan?

Las prácticas de revisión, control y, en un lenguaje más contemporáneo, evaluación de los actos del gobierno son de larga data en el desarrollo institucional chileno (Olavarría Gambi, 2008). Las primeras prácticas fueron más bien formales, apegadas a criterios contables y legales -como eran las recomendaciones de la época-, pero a medida que fue avanzando el desarrollo conceptual, metodológico y disciplinario de las Ciencias Sociales las prácticas se fueron sofisticando, dando paso a la noción de evaluación como una práctica diferenciada de las actividades de fiscalización y control ${ }^{4}$.

Visto en perspectiva $-\mathrm{y}$ sin perjuicio de la diferenciación entre fiscalización y control, por un lado, y evaluación, por otro- lo que muestra el desarrollo institucional chileno es una constante preocupación por el correcto uso de los recursos públicos. En los inicios la expresión "correcto uso" estuvo asociada al apego a la legalidad y, luego, el avance de las ciencias sociales y su instrumental ha ido adicionando prácticas de evaluación a lo largo del proceso de políticas públicas. No obstante, la aplicación de estos principios a los distintos ámbitos de intervención del gobierno es desigual.

La presencia/ausencia de evaluación en las políticas públicas bien podría entonces operar como indicador del grado de madurez en la formulación de las mismas, actuando entonces como baremo sobre la propia capacidad del sistema político de poder asegurar una mayor racionalidad en la aplicación de las mismas, y la propia posibilidad también del conjunto de la sociedad de poder ponderar con mayor atención lo que los gobiernos hacen en su nombre. Y con su dinero.

De modo que un Estado que desarrolla acciones pero no las evalúa (o al menos no presenta a la comunidad el resultado de las evaluaciones operativas que realiza) no desarrolla en propiedad "políticas públicas". Un Estado que no da cuenta de los resultados de sus balances a la comunidad, tampoco estaría cumpliendo a cabalidad con la exigencia democrática de

\footnotetext{
${ }^{4}$ La primera práctica de revisión de los emprendimientos públicos, relata Olavarría Gambi (2008), data de 1541 cuando, luego de la fundación de Santiago, el primer Cabildo designó a una comisión compuesta por cuatro oficiales con la misión de supervigilar el correcto uso de los fondos del reino.
} 
transparencia y responsabilidad que se exige hoy por hoy a las sociedades políticamente organizadas.

Es de suyo conocido que el Estado chileno realiza una acción cultural concreta desde los inicios de la vida republicana del país. ¿Es posible reconocer en esa experiencia histórica algunas condiciones que nos permitan caracterizar mejor la conformación de las políticas culturales en Chile durante el siglo XX?, ¿podría servir la presencia/ausencia de evaluación como un factor discriminador del calado de las intervenciones públicas en el desarrollo cultural del país?

A fin de cuenta, lo que nos interesa conocer es si son las acciones del Estado chileno auténticas "políticas públicas en cultura", al menos como las hemos descrito en la sección precedente.

\section{ETAPAS EN EL DESARROLLO DEL ACCIONAR CULTURAL DEL ESTADO CHILENO}

Hay que aclarar de primeras que no es la perspectiva historiográfica el más popular de los enfoques de análisis entre los especialistas en políticas culturales. Algunos trabajos disponibles establecen comparaciones entre la evolución institucional en países cercanos en el centro de Europa (Negrier, 2003), otros afincados en nuestro subcontinente enfatizan una perspectiva más cercana a la arqueología del derecho cultural y administrativo (Harvey, 2006, 2008). La mayoría de los aportes disponibles ahondan sin embargo, en la reconstrucción de las políticas culturales en Francia, especialmente a partir de la creación en 1959 del Ministerio de Cultura por el gobierno de De Gaulle (Baker, 1987; Beaulieu, 2002).

Para la experiencia chilena, las aproximaciones sistemáticas son aún más escasas. Los meritorios aportes de Karina Arias (Arias Yurisch, 2010; Arias Yurisch \& Gálvez Gómez, 2010), incluyen referencias interesantes sobre la evolución de nuestras instituciones culturales y el testimonio biográfico de algunos de los protagonistas de procesos centrales de su conformación en las décadas pasadas. Squella (2005) aporta también una perspectiva más personal al análisis. Otros aportes, como los de Subercaseaux (1991, 1993, 1997) han estado referidos al estudio de algunas prácticas culturales definidas (vg. el libro y la lectura).

Pero carecemos de visiones de conjunto sobre la evolución de las políticas culturales chilenas, no disponemos tampoco de miradas que permitan comparar, aun con mayor perspectiva, los aspectos centrales de ese proceso na- 
cional en el contexto del desarrollo disciplinario del tema, tanto en el plano latinoamericano como en su proyección a las sociedades de corte occidental. Siguiendo, con algunas licencias, los ciclos que el especialista argentino Edwin Harveys ha identificado en numerosos trabajos sobre las políticas culturales, el derecho cultural y la acción cultural de los estados americanos $^{5}$, nos parece reconocer en esta experiencia bicentenaria de la intervención cultural del Estado chileno, tres etapas más o menos perfiladas, cada una conteniendo a su vez distintas fases (Ver Tabla 1).

Tabla 1. Etapas de desarrollo de las políticas culturales en Chile, 1810-2010.

\begin{tabular}{|c|c|c|}
\hline $\begin{array}{l}\text { Etapas de desarrollo de las } \\
\text { políticas culturales }\end{array}$ & Subperiodo & $\begin{array}{l}\text { Características } \\
\text { relevantes }\end{array}$ \\
\hline \multirow[t]{3}{*}{$\begin{array}{l}\text { Etapa de la acción cultural } \\
\text { del Estado } \\
1810-1960\end{array}$} & $1810-1910$ & $\begin{array}{l}\text { Etapa fundacional. Labor civilizadora del } \\
\text { Estado, educación popular, básica, obligatoria. } \\
\text { Patrimonialismo y mecenazgo estatal. }\end{array}$ \\
\hline & $1910-1930$ & $\begin{array}{l}\text { Creación de primeros organismos administrativos } \\
\text { nacionales de fomento y promoción de la cultura. } \\
\text { Mecenazgo estatal. }\end{array}$ \\
\hline & $1930-1960$ & $\begin{array}{l}\text { Democratización de la cultura en contexto de } \\
\text { polarización política. Extensionismo. Cultura } \\
\text { como subsector de Educación. Inicio de industrias } \\
\text { culturales, desarrollo de politics. Mecenazgo } \\
\text { estatal. }\end{array}$ \\
\hline \multirow[t]{3}{*}{$\begin{array}{c}\text { Etapa de las políticas } \\
\text { culturales } \\
1960-2010\end{array}$} & $1960-1970$ & $\begin{array}{l}\text { Democratización de la cultura en contexto de } \\
\text { polarización política. Basismo. Cultura como } \\
\text { subsector de Educación. Cultura como tema } \\
\text { ineludible para el Estado, primeras "políticas } \\
\text { culturales" en forma. }\end{array}$ \\
\hline & $1970-1990$ & $\begin{array}{l}\text { Época de profundos cambios, desde extrema } \\
\text { politización en los } 70 \text { 's hasta concepciones más } \\
\text { tecnocráticas en los } 90 \text { 's. Cultura como subsector } \\
\text { de Educación. }\end{array}$ \\
\hline & $1990-2010$ & $\begin{array}{l}\text { Organismos centralizados de administración } \\
\text { cultural del Estado (CNCA), concursabilidad para } \\
\text { la asignación de recursos, leyes de donaciones } \\
\text { modales, subsidio a la oferta, transferencias } \\
\text { directas. Balances. Gran desarrollo legislativo para } \\
\text { el sector. }\end{array}$ \\
\hline $\begin{array}{l}\text { Etapa de las políticas } \\
\text { públicas en cultura } \\
2010 \text { ¿? }\end{array}$ & 2010 & $\begin{array}{l}\text { Industrias creativas, desarrollo de cultural } \\
\text { public policies, leyes de mecenazgo, subsidio a la } \\
\text { demanda. Accountability. Evaluación Integral. }\end{array}$ \\
\hline
\end{tabular}

Fuente: Elaboración propia.

${ }^{5}$ Director de la Cátedra UNESCO de Derechos Culturales, Universidad de Palermo, Buenos Aires y ex director del Fondo Nacional de las Artes (1983-89), el abogado, docente e investiga- 
A. Etapa de la acción cultural del Estado (Government's cultural action) 1810-1960

Por "acción cultural del Estado" entiendo aquí aquel conjunto de decisiones que los gobiernos asumen bajo la premisa dominante de contribuir a la "civilización de la población" y a la transmisión de los "valores culturales", donde su eje dominante de actuación es la defensa y cuidado del patrimonio; orientado desde y para las élites, con especial vocación educadora, donde las universidades públicas asumen un papel relevante como órganos de difusión cultural.

No existe, más allá de la comercialización en circuitos reducidos, un mercado de bienes y servicios culturales. De hecho la producción cultural se mantiene ajena a consideraciones de orden económico; el financiamiento de las instituciones se alcanza por asignaciones directas contenidas en el presupuesto general de la Nación o, en casos individuales, por el subsidio personalizado al artista bajo la figura de una "beca presidencial" o, en la mayoría de los casos, por su asimilación como "funcionario público". El principal mecenas es el Gobierno. No hay leyes definidas para el sector, más allá de los reglamentos y decretos fundacionales con lo que se crea la Universidad del Estado o de la Nación, el Liceo Nacional, el Museo Nacional, el Teatro Nacional y, entre otros, el Conservatorio Nacional.

El espacio cultural arquetípico es el Ágora de la Universidad, el Ateneo de las artes y la sala principal del Teatro Nacional. A ellas concurren preferentemente los artistas "institucionalizados", las élites económicas y sociales y los intelectuales.

El estilo extensionista de la acción cultural (Rubinich, 1993) configurará a la postre una práctica más vinculada con la educación para las artes, la

dor Edwin Harvey es profesor y director de cursos de grado y postgrado en derecho, política y gestión cultural en universidades argentinas y extranjeras, a partir de la dirección del Primer Curso Interamericano de Administradores Culturales (OEA, Caracas, 1975). Autor de una amplia bibliografía especializada sobre política cultural, financiamiento de la cultura, relaciones culturales internacionales y derecho de la cultura, publicada en Buenos Aires, Bogotá, San Juan de Puerto Rico, Madrid y París, entre 1975 y 2007. Últimas obras: Financiación de la Cultura y de las Artes (Iberoamérica en el contexto internacional), Madrid, 2003; Política y financiación pública de la cinematografía, Madrid, 2005; Política y financiación pública del teatro, Madrid, 2005; Política y financiación pública de la música, Madrid, 2006. En materia de políticas culturales y de derechos culturales son de señalar las obras: Políticas Culturales en Iberoamérica y el mundo, Madrid, 1990, y Derechos culturales en Iberoamérica y el mundo, Madrid, 1990. 
propaganda del régimen y la promoción de los talentos individuales. Su objetivo fundamental era difundir la cultura entre los ciudadanos independientemente de su posición social, sexo o adscripción territorial. La cultura dejaría así de ser propiedad exclusiva de las élites y se democratizaría, facilitándose el acceso a la misma. Para ello se pone en circulación todo el patrimonio cultural acumulado en épocas anteriores. Las estrategias de ayuda a la creación, producción y sobre todo a la difusión serán las dominantes en esta época, caracterizada por las contradicciones y la lucha ideológica.

a.1. Un primer momento para el ulterior desarrollo de la acción cultural del Estado está situado a partir de los albores de la República (1810) y se extiende hasta 1910 aproximadamente. Período largo que sorprende al Estado asumiendo que parte fundamental de su labor "civilizadora" comprendía expandir la cultura y formar el "gusto" a través del arte. Allí está el empeño de los fundadores de la República para levantar la Universidad de Chile (1842), el Instituto Nacional (1813), el Teatro Municipal de la ciudad capital (1857), el Conservatorio de la Música (1850), el Archivo Nacional (1927) entre tantas obras que nos hablan de ese empeño fundacional que nos legó el patrimonio institucional del que hoy gozamos.

Durante las primeras décadas del siglo XIX, su labor de mecenazgo entregó un importante apoyo económico y un subsidio directo en el campo -principalmente de las artes-, que, podríamos decir, caracterizó casi completamente las primeras aproximaciones a una política cultural más o menos consciente por parte del Estado. Pero se trataba aún de acciones puntuales, especialmente en lo referido al proceso fundacional de grandes instalaciones culturales como la Biblioteca Nacional, el Archivo Nacional, los primeros museos nacionales y algunos grandes teatros públicos.

Este largo segmento está marcado por la presencia casi omnímoda del Estado y sus órganos de expresión.

En parte por la propia inercia que el Estado traía desde mediados del siglo XIX, en parte por una concepción bastante extendida por aquel entonces que le entregaba al sector público un papel preponderante en la economía y otros planos de la vida social; fue característico, hasta bien entrados los 50 del siglo XX, el protagonismo estatal como principal animador de la vida cultural del país. La realidad chilena no es sino el reflejo de una tendencia que se apreciaba además allí donde se dirigiera la mirada en el subcontinente.

Estatales eran las universidades, la educación básica y secundaria en su mayor extensión, los medios de comunicación más importantes. La pro- 
ducción individual más destacada era en el ámbito de la poesía (Mistral, Neruda, Huidobro, entre otros) y en la historia (Góngora, Eyzaguirre, Encina).

Institucionalmente hacia fines del siglo XIX y principios del XX existía un esquema general caracterizado por la no existencia de una política cultural coherente por parte del Estado. Hay sí acciones puntuales en distintos planos de la vida cultural, especialmente en lo referido al proceso fundacional de grandes instalaciones culturales.

a.2. Un segundo momento Harvey lo sitúa para América Latina en la transición de los años treinta, en que aparecen organismos administrativos nacionales de fomento y apoyo a la cultura de carácter permanente y las actividades se dirigen tanto a la protección del patrimonio histórico como del artístico. La administración de estos soportes institucionalizados es empírica, está generalmente a cargo de personas a quienes el Estado quiere distinguir por sus méritos intelectuales.

Existe además una fuerte actividad de mecenazgo cultural por parte de la burguesía local, son las familias quienes financian las acciones culturales concretas. La cultura se concibe como un apéndice de la educación, tanto conceptual como administrativamente. La promoción del libro y la lectura que aparecen en esta época son concebidas como una forma específica de la lucha general contra el analfabetismo.

Hay una abundante legislación sobre políticas culturales, leyes sobre patrimonio, archivos, propiedad intelectual, derechos de autor, reconocimientos y premios a artistas e intelectuales. En 1933 la Conferencia de Montevideo trata por vez primera el tema del patrimonio cultural, al reconocer el usufructo pero no la propiedad de los bienes patrimoniales (Prieto de Pedro, 2009).

La generación de organismos administrativos de fomento y apoyo a la cultura de carácter permanente, especialmente aquellos que se dirigen tanto a la protección del patrimonio histórico como a las creaciones del ámbito de las artes, las ciencias y las humanidades (por ejemplo en Chile la Dirección de Bibliotecas, Archivos y Museos -DIBAM- creada en 1929), constituye un hito en nuestra breve historia de las políticas culturales.

a.3. Un tercer momento es el que sitúa en el contexto de la posguerra y la década de los cincuenta, en que se crean las primeras Organizaciones Intergubernamentales de carácter mundial alrededor de las Naciones Unidas. Se acentúa la acción internacional en Educación y Cultura, como también el 
apoyo y la preocupación por el papel cultural de las industrias culturales (principalmente radio, televisión, periodismo y cine).

Al concluir la Segunda Guerra Mundial y comenzar a difundirse el concepto de "derechos culturales" (Achugar, 2003; Carmona Tinoco, 20022003) inscrito en la Carta de las Naciones Unidas, se acentuó la necesidad de la acción estatal en los ámbitos de la Educación y Cultura, como también el apoyo y la preocupación desde el Estado, por el papel cultural de las nacientes industrias de la entretención (principalmente radio, televisión, el periodismo y el cine) ${ }^{6}$.

La acción cultural del Estado se ve fuertemente influenciada por consideraciones de orden ideológico, pasando la actividad cultural a ser referida más que nada por su capacidad de portar contenidos políticamente comprometidos. Es la era de las politics.

El principio dominante de la democratización cultural se fundamentaba en la práctica de las acciones culturales de los gobiernos de los años inmediatos a la Segunda Guerra. Basado en el apoyo a la creación, el desarrollo de infraestructura para la producción cultural, el mantenimiento de altos estándares de calidad, en la dimensión profesional de la actividad cultural y en las formas de expresión artística más reconocidas para hacerlas accesibles a la población; la acción pública desarrollada bajo este enfoque tendió a ser más centralizada.

\footnotetext{
${ }^{6}$ ¿Cuáles son entonces los llamados Derechos Culturales? Por "derechos culturales" se suele aludir específicamente al derecho a la educación, el derecho a la participación en la vida cultural; el derecho a gozar de los beneficios del progreso científico y de sus aplicaciones y el derecho a beneficiarse de la protección de los intereses morales y materiales emergentes de la producción científica, literaria y artística de que sea autor. Poco después, y bajo el amparo de la UNESCO, una serie de encuentros mundiales realizados en cada continente permitió precisar de mejor forma las implicancias del desarrollo cultural, la cooperación cultural internacional y el derecho a la cultura. En la reunión de Venecia por ejemplo, en 1970 se declaraba que: “... debe hacerse todo lo posible para crear las condiciones económicas y sociales que permitan el acceso libre y democrático a la cultura"; mientras en Helsinki, en el '72, se recomendaba a los estados de Europa que garantizaran el derecho a la cultura y el libre acceso a los tesoros de la cultura nacional y universal de todos los miembros de la sociedad. En Yogyakarta, en 1973, se sostenía que: “... para garantizar a la población el derecho a la cultura y proporcionarle el acceso libre y democrático a ella, es indispensable que los poderes públicos elaboren y apliquen una política cultural clara y consecuente orientada hacia esos objetivos" y en Bogotá, en 1978, los delegados concluían que los derechos culturales deben ser el elemento básico del programa de la UNESCO sobre la cultura.

Fue precisamente en la reunión de México, en1982, durante la última y más grande Conferencia Mundial sobre Políticas Culturales, que se señaló la necesidad de la más amplia participación del individuo y la sociedad en la creación de bienes culturales, en la toma de decisiones que conciernen a la vida cultural y en la difusión y disfrute de la misma. La conferencia mexicana tiene desde mi perspectiva el mérito adicional de abrir una nueva etapa para la reflexión sobre las políticas culturales, pues de ella se deduce que son las políticas públicas en cultura (arts and cultural public policies) las encargadas de operativizar (llevar a la práctica) el Derecho a la Cultura que asiste a cada ciudadano.
} 
Hasta fines de los años cincuenta, esta política comenzó a ser sustentada en América Latina por Estados e instituciones culturales, que priorizaron la difusión y la popularización de la alta cultura con el objetivo de lograr el acceso de todos los individuos y grupos al disfrute de los bienes culturales.

Naturalmente no existe evaluación de las mismas, más allá de la formalidad de los juicios políticos y el cumplimiento de las metas presupuestarias. Pese a que han comenzado su desarrollo disciplinar tanto en los Estados Unidos como en Europa meridional, la teoría de las políticas públicas aún vive una etapa de desarrollo incipiente en América Latina.

B. Etapa de las políticas culturales (Government's cultural politics) 1960-2000

La que concebimos aquí como "etapa de las políticas culturales de Gobierno" coincide con un remozado interés de los gobiernos por el vínculo entre cultura y educación, aunque el sector objetivo de los beneficios fiscales en cuanto a financiamiento y promoción legal seguirá siendo, por una parte, las élites artísticas y la comunidad escolar. Las universidades del Estado continuarán actuando, al menos por un tiempo, como "faros" desde donde se ilumina la creación individual y la generación de vanguardias, pero irán perdiendo lenta e inexorablemente su protagonismo en la misma medida en que se politizan y asumen discursos sobreideologizados que los distancian del ciudadano medio y se ven sometidas a tensiones presupuestarias que reducirán sus presupuestos.

Los equipamientos más habituales de intervención serán las bibliotecas públicas, los museos y las recién estrenadas "casas de la cultura", que como propuestas polivalentes de acción cultural estas últimas, asumirán indistintamente el carácter de focos de animación sociocultural (allí cuando más cerca estén de las bases) o de administración cultural (allí cuando más cerca estén del mercado).

Esta tensión manifiesta, entre intervención política e intervención comercial, desembocará en la configuración de "modelos" más o meno reconocibles de políticas culturales de Estado (Bodo, 2004).

Las industrias culturales se encuentran ya plenamente integradas a mercados globales, sometidas por cierto a las mismas tendencias que afectan a las actividades comerciales, aunque los estados continúan con los subsidios a la oferta artística, generando las primeras leyes para su promoción como industrias protegidas. El mecenazgo del Estado cede su espacio a crecientes formas de cooperación con la empresa privada y los particulares, ya sea 
bajo la forma de regímenes de mecenazgo con causa modal o donaciones con fines particulares (Antoine, 2005).

El estilo dominante de las políticas culturales será el "basismo", es decir, se apelará a una definición más amplia de la cultura (UNESCO, 1982), se defenderá la diversidad de formas de expresión y su integración a la vida cotidiana, suponiendo un modo de intervención más descentralizado, que favorece una más amplia participación de los ciudadanos a la vida cultural y un crecimiento de su capacidad de decisión.

b.1. Desde fines de los sesenta y hasta fines de los setenta, en América Latina esta política tuvo como principales agentes a los partidos progresistas y los movimientos populares independientes. A través de la promoción de la participación popular y la organización autogestionada de las organizaciones culturales y políticas, se proponía el desarrollo plural de las culturas de todos lo grupos en relación con sus propias necesidades.

Será en la década de los setenta donde el tema y el problema cultural se convierta en ineludible. La discusión se inicia con la importante Conferencia de Venecia sobre políticas culturales que, dentro de sus recomendaciones, llama a los países a realizar reuniones continentales para esclarecer una posición ante las políticas culturales regionales (Harvey, 2007).

Las acciones internacionales se incrementan en forma relevante y se vincula la dimensión cultural a políticas de planeación. Por ejemplo, en Bogotá, Colombia, en 1978, se realiza la Conferencia Intergubernamental sobre políticas culturales para América Latina y el Caribe, que postula destacadas recomendaciones para los países de la región. La conferencia se dedica a reconsiderar la opción por el desarrollo desde una nueva óptica y el tema de los derechos culturales cobra cada vez mayor vigencia.

La cultura no estará ajena a estos intentos de planificación centralizada, de modo que las instituciones antes dispersas sufren un proceso concentrador siguiendo el modelo que con tanto ahínco proclamaba Malraux desde la capital gala.

Tres funciones básicas tienen estos nuevos entes centralizadores de la cultura: Bellas Artes, conservación del patrimonio y difusión cultural. De modo que mientras el aparato estatal se institucionalizaba en los referentes más elitistas de la cultura, los movimientos de izquierda reivindicaban como arquetipo propio "lo popular" (Hunter, 2005).

Los setenta estarán caracterizados también por el activismo, el trabajo empírico, intuitivo y voluntarista (Gillet, Jeannel, \& Ion, 1995). Aparece por estos años el 'animador cultural' emparentado con modelos europeos de 
activismo político de izquierdas. Hasta comienzos de esa década la cultura era considerada como algo ajeno a la sociedad, se la mantenía aparte, encerrada en las salas de exposiciones y en las galerías de los museos decimonónicos. A partir de estas reuniones internacionales, no obstante, la cultura se asumirá como una responsabilidad del Estado.

b.2. En este periodo el sector público será el encargado de incorporar el factor cultural en los planes de desarrollo de las sociedades nacionales y, por lo mismo, el responsable igualmente de planificar la acción cultural concreta. Claro que lo hará en un contexto de fuerte agitación política y confrontación ideológica. Las décadas de los ochenta y noventa también serán testigo de la evolución que se produce al interior de la profesionalidad cultural. El activista, animador, militante de la vida cultural, deberá ir cediendo paulatinamente su espacio a un administrador profesional de entes culturales.

Desde comienzos de los setenta se concibió en Chile a la cultura como un instrumento de carácter ideológico, vector fundamental de la identidad revolucionaria que se impulsaba desde el Ejecutivo, empeñado en hacer "una revolución en libertad". La cultura fue entendida como un instrumento transformador de las conciencias que buscaba desarticular el ordenamiento cultural burgués para reconstruirlo desde la base de los intereses de las clases populares.

Los cursos de acción de la gestión cultural del gobierno de la Unidad Popular (UP) (Catalán, Guilisasti, \& Munizaga, 1987) estuvieron caracterizados además por la existencia de una estrategia de apropiación directa por parte del Estado de las empresas productivas que operaban en el área cultural; también por iniciativas destinadas a apoyar las actividades culturales de las organizaciones populares y el intento de crear un control indirecto de las actividades en manos de la empresa privada para incidir en la dirección de la acción cultural. No estuvieron ausentes también las iniciativas destinadas a apoyar las actividades culturales de las organizaciones populares y el intento de crear un control indirecto de las actividades en manos de la empresa privada, en un esfuerzo por incidir en la dirección de la acción cultural (Bowen Silva, 2008).

A comienzos de esa década, grupos de intelectuales de la Unidad Popular (Subercaseaux, 2006), señalaban que la cultura debía alcanzar los privilegios necesarios para establecer la utopía global de un "hombre nuevo". Uno de sus objetivos era crear un Ministerio de la Cultura, con lo que se reorientarían y resolverían las contradicciones culturales de Chile.

Asimismo, buscaban formar una institución que pudiese desarrollar la 
actividad cultural del país, crear nuevos espacios, fomentar el sector cultural público y privado y dar solución a las necesidades de la sociedad. Predominaba la idea de crear una cartera dedicada exclusivamente a los asuntos culturales, buscando un instrumento para desarrollar lo que se entendía como una de las bases fundamentales de la nueva sociedad que planteaba el gobierno socialista de Salvador Allende.

La intervención militar de septiembre de 1973 puso fin a esta dinámica. Aunque no interrumpió totalmente la actitud del Estado de considerarse como un actor principalísimo de la misma. El Gobierno Militar no se alejó significativamente de los esfuerzos desplegados por otros gobiernos por consolidar una institucionalidad cultural pública distintiva que asegurara al Estado un rol de preeminencia en este ámbito.

Prueba de ello es su papel en la organización del Departamento de Extensión Cultural, antecesor de la División de Cultura en el Ministerio de Educación, a la postre su principal instrumento de intervención durante décadas. Creó la Dirección de Asuntos Culturales del Ministerio de Relaciones Exteriores para asegurar la difusión de sus actividades en el exterior a través de las agregadurías culturales en el extranjero; fundó casas de cultura y corporaciones culturales en las principales municipalidades del país y, ProChile, un organismo técnico destinado a promover las exportaciones y la "imagen país" que ha descansado tradicionalmente en el soporte cultural de algunas industrias creativas.

El régimen militar impuso legalmente a los canales de televisión disponer una "franja cultural" obligatoria a la semana y, entre otras iniciativas, realizó anualmente unas Jornadas Nacionales de Cultura. Este empeño institucionalizador contrasta (Cavallo, Salazar, \& Sepúlveda, 2001) con la persecución de artistas e intelectuales por sus ideas políticas y la implantación de un férreo círculo de censura.

El predominio de políticas económicas de corte neoliberal que se fueron imponiendo paulatinamente en la conformación del modelo económico chileno desde fines de los setenta, supuso una paulatina disminución de los subsidios directos hacia el sector cultural (Stanziola, 2002), poniendo en evidencia, una vez más, que las políticas culturales no son independientes de la existencia de otras políticas influyentes, como suelen serlo las políticas tributarias o de hacienda.

Uno de los efectos probablemente no deseados por la implantación de estos cambios estructurales en la forma de financiamiento de la actividad cultural fue el denominada "apagón cultural" (Collyer, 1986; Henríquez Moya, 2003) que afectó a las artes y las humanidades en Chile. Con el tiem- 
po, el mercado pasó a jugar un rol que nunca antes tuvo en la historia de Chile (Polle \& Fernández Jilberto, 1992). A su sombra aparecieron nuevas formas de financiamiento de la cultura, entre ellas el mecenazgo del sector privado (Antoine, 2005).

\section{b.3. A la postre el régimen militar impuso una política cultural caracterizada} por el dirigismo en lo político y el liberalismo en lo económico. La última década del siglo fue testigo del reconocimiento de que las políticas públicas en Cultura deberían adecuarse a la idea de una mayor prescindencia del Estado en materias culturales. El retorno a la democracia en Chile en 1990, sin embargo, pondría un acento renovado a la presencia estatal en lo cultural. Los gobiernos democráticos diseñaron, a su vez, su propia estrategia de intervención estatal en la cultura, guiados por algunos criterios básicos. Entre ellos el garantizar la libertad de creación y de expresión; aceptar la autonomía de los procesos culturales frente al Estado; favorecer la equidad en el acceso a la cultura; estimular la participación de todos los sectores en la vida cultural; reconocer (fáctica y legalmente) la diversidad de culturas y de identidades étnicas dentro del país; fomentar la descentralización en la producción y gestión culturales y cumplir con el deber del Estado de proteger y difundir el patrimonio físico y espiritual de la Nación.

A partir de los años ochenta y hasta el cambio de siglo, son destacables tres aspectos: la discusión por el papel y límites del Estado en la formulación de las políticas culturales; la preocupación por un incremento cualitativo en la participación de la sociedad en su conjunto y el papel destacado de la comunidad universitaria en las acciones relativas a su formulación (David Garson, 1992).

En América Latina aparece una nueva concepción cultural representada además por la expresión "dimensión cultural del desarrollo". La idea, enunciada por la UNESCO en México en 1982 anuncia un nuevo modelo de política cultural. Ya no se trata tan solo de conservar la cultura y ponerla al acceso de la gente, sino también de reconocer lo popular en la creación cultural; existe entonces un movimiento ascendente que sobrepasa al mismo creador, factor que necesariamente reclama otro tipo de espacio y profesional.

El déficit institucional asociado a los desajustes de gestión en las políticas culturales latinoamericanas caracterizarán la década de los ochenta. Por su parte, la crisis económica afectó principalmente a las industrias culturales, en particular a las que dependían del Estado. La pérdida de consistencia de todos los aparatos creados por el Estado para administrar la cultura cerró 
bruscamente un período de casi medio siglo de intervencionismo público en materias culturales.

Así, por una parte, veremos desde los años ochenta en adelante, la discusión por el papel y límites del Estado en la formulación de las políticas culturales; la preocupación por un cualitativo incremento en la participación de la sociedad en su conjunto y el papel destacado de la comunidad universitaria en las acciones relativas a su formulación, corre casi en paralelo con el desarrollo de las metodologías y estrategias para la "medición" de la eficiencia y la eficacia de las decisiones gubernativas en tal sentido.

Los años noventa estuvieron caracterizados por la crisis de la institucionalidad cultural en el subcontinente, pues no ha sido ajeno en nuestros países el discurso en estos últimos años favorecedor del planteamiento de nuevas formas de institucionalidad de la cultura ${ }^{7}$.

La última década del siglo pasado fue testigo del reconocimiento de la existencia de mercados abiertos y competitivos que han obligado a adecuar la doctrina cultural en boga en este último tercio de la centuria a la idea de una mayor prescindencia del Estado en materias culturales. Asume ahora el Estado un papel más activo en lo que se denomina el patrimonio colectivo (Cultura y medio ambiente), en el convencimiento de que a la población de un Estado (sus ciudadanos) lo que los mantiene unidos es ese vínculo por el que devienen en comunidad. Y ese vínculo es cultural.

Con la creación en 2003 del Consejo Nacional de la Cultura, el gobierno de Ricardo Lagos pretendió asegurar una coordinación permanente entre todos aquellos organismos del Estado chileno que tenían ascendencia en materias culturales. En efecto, a poco de iniciado su mandato, el presidente Lagos dispuso personalmente la instalación de una coordinación

\footnotetext{
${ }^{7}$ Entre ellos destaca la adopción de mecanismos financieros: subsidios de mantención de infraestructura y patrimonio (bibliotecas, archivos, museos), créditos (cine), exenciones tributarias (Ley de Donaciones). Medidas de regulación del mercado cultural: a través de leyes que amplíen la participación y favorezcan el desarrollo de sectores artísticos e industriales específicos (Ley de Propiedad Intelectual, del Libro y la Lectura, etc.). Incentivos o estímulos directos: a través de premios, becas, fondos especiales (Premios nacionales, Fondo de Desarrollo de las Artes y la Cultura; Fondo de Apoyo a Iniciativas Culturales Regionales, Fondo del Libro y Lectura, Fondo de Desarrollo Artístico Cultural para establecimientos de enseñanza artística, Fondo de Desarrollo Científico y Tecnológico). Prestación de servicios en forma directa: extensión cultural a través de BAFONA, Orquesta de Cámara de Chile, Orquesta Sinfónica Juvenil, Teatros Itinerantes. Reordenamiento administrativo: Articulación entre los niveles local, regional y nacional (bibliotecas, SEREMIS, universidades). Mecanismos internacionales: convenios y tratados de integración y cooperación, para coproducciones, ampliación de mercados, difusión e intercambio (DIRACI, PROChile, Oficina de Relaciones Internacionales del MINEDUC).
} 
permanente de los organismos gubernamentales que cumplen funciones en el ámbito cultural y que encontraban bajo la dependencia de distintos ministerios, tales como División de Cultura del Ministerio de Educación, Dirección de Bibliotecas, Archivos y Museo, Dirección de Relaciones Culturales del Ministerio de Relaciones Exteriores. Departamento de Cultura del Ministerio Secretaría General de Gobierno y Consejo de Monumentos Nacionales. Esta sería la base de la nueva institucionalidad pública de la cultura ${ }^{8}$.

A partir de su creación, el CNCA ha implementando políticas culturales públicas que recibieron un impulso sustantivo durante la administración de Paulina Urrutia, la actriz independiente al frente del organismo. Buena parte de las tareas desempeñadas ha estado dominada por un afán todavía institucionalizador, en el sentido de continuar creando los organismos y leyes que dieran sustento a la tarea cultural del Estado chileno.

De allí los esfuerzos (frustrados unos, exitosos otros) de avanzar en la renovación de la legislación de protección del patrimonio, la creación de un Instituto del Patrimonio, un Plan Nacional de Turismo Cultural, la Cineteca Nacional, las Bases de Datos y la Cuenta Satélite de Cultura. Se han entregado más recursos a los Consejos Regionales de Cultura; se fomentó a la educación artística, se impulsó la inserción de gestores culturales en el Servicio País y se diseñó un plan para dotar a las comunas de más de cincuenta mil habitantes con un centro cultural o una biblioteca.

En el plano de las políticas culturales, el documento Chile Quiere más Cultura propuso las definiciones de política cultural para el período 20052010 y fue el fruto de un esfuerzo colectivo encabezado por el directorio nacional del Consejo Nacional de la Cultura y las Artes (CNCA), que comprometió la participación de los trece Consejos Regionales y de los Comités Consultivos. A la postre, el documento consagró como "política cultural"

\footnotetext{
${ }^{8}$ La idea de una entidad de control de las organizaciones culturales del Estado no era original, sin embargo, del tercero de los gobiernos de la Concertación de Partidos por la Democracia, la alianza política que derrotó a Pinochet en las urnas. La puesta en operaciones de un organismo público que coordinará la actividad cultural del Estado al más alto nivel era aspiración antigua para la coalición política que gobernó al país hasta marzo de 2010. Un organismo de similares características aparecía ya en el programa de gobierno de Patricio Aylwin (1990-1994), a comienzos de los 90, cuando se destacaba la necesidad de configurar una instancia coordinadora que permitiera conducir con mayor coherencia y eficacia las políticas y acciones del sector público en el campo cultural. En efecto, el programa de Gobierno del propio Aylwin proponía estudiar la configuración de una instancia coordinadora u organismo superior, que permita conducir con mayor coherencia y eficacia las políticas y acciones del sector público en el campo cultural.
} 
del Estado chileno a nueve principios ordenadores, cuatro líneas estratégicas, diez objetivos centrales y 52 medidas específicas?.

Las cifras dan cuenta además de la atención puesta por las autoridades al desarrollo artístico y cultural. Entre 2006 y 2010 se triplicó el presupuesto, se pasó de 22 mil a 63 mil millones de pesos.

La inversión permitió que 6 mil proyectos fuesen financiados, gracias a las políticas generadas por el Consejo Nacional de la Cultura y las Artes $(\mathrm{CNCA})^{10}$. La gestión de la institución logró el perfeccionamiento y mejoramiento de los procesos de concursabilidad y la asignación de los recur$\operatorname{sos}^{11}$.

En síntesis, con la sola excepción del interregno democrático que fue el gobierno militar para la cultura en al menos parte significativa de su administración, el Estado chileno ha sido permanentemente un actor decisivo en la formulación de políticas culturales a lo largo del último cuarto de siglo. Con algunas inflexiones coyunturales, ha sido además un factor de influjo poderoso, especialmente en el financiamiento de las actividades y la promoción de las mismas a través de una serie de instituciones de su dependencia con incidencia en el desarrollo del sector. A ese espacio de la presencia orgánica (estructuras y aparatos) y jurídica (leyes reglamentos) del Estado en cultura se le denominó desde mediados de los 90 "institucionalidad cultural" (Squella, 2006).

Concepto que en un sentido restringido de la expresión está constituido por los organismos gubernamentales que cumplen funciones en el ámbito de la cultura. Pero que, en un sentido amplio, comprende las políticas cul-

\footnotetext{
${ }^{9}$ De todas las medidas dispuestas solamente un 23\% presentaban a marzo de 2010 un nivel de cumplimiento total y otro $23 \%$ llegaban a un nivel de cumplimiento alto. Dicho más directamente, sólo 24 medidas llegaron, después de cinco años de implementación, a un nivel observable de cumplimiento. De las recomendaciones hechas al CNCA por la Dirección de Presupuesto el 2008, sólo se habían cumplido el $18 \%$ a la fecha indicada. Cfr. Cuenta Pública CNCA, agosto 2010 , pp. 5 y 6.

${ }^{10}$ No obstante, los resultados de la 2a Encuesta Nacional de Participación y Consumo Cultural en que se analizaron 4.176 casos durante enero y abril de 2009, con una cobertura en las 15 regiones del país, puso nuevamente en evidencia la brecha entre la Región Metropolitana y el resto del país, aunque un $85 \%$ de la población cree que tiene más acceso a la cultura que sus padres, y el 66,7\% piensa que los bienes culturales son más accesibles que hace cinco años (Abarca Besares, 2009). Más preocupante es que a un tercio de los chilenos les da lo mismo vivir en dictadura o en democracia.

${ }^{11}$ Los aumentos en la cobertura y la febril actividad que el CNCA desarrolló en estos últimos años fueron opacados, empero, por denuncias públicas de errores gruesos en la gestión administrativa de la entidad, abusos de confianza y preterición por criterios políticos -que terminó incluso con el proceso abierto a algunos de sus máximos directivos por los presuntos abusos cometidos.
} 
turales públicas en aplicación; los organismos que definen tales políticas y que las aplican por medio de los programas y proyectos que ejecutan; el personal calificado a cargo de la gestión de esos organismos; los presupuestos públicos de que se dota a éstos y con los que se retribuye y capacita a dicho personal; los instrumentos por medio de los cuales se asignan recursos públicos a la cultura, tales como fondos concursables y otros; los estímulos para la inversión privada en cultura; y las disposiciones constitucionales, legales y reglamentarias que dan soporte normativo a cada uno de los aspectos antes señalados.

Con respecto a la evaluación de las políticas públicas, sabemos que antes de 1973 éstas tenían su origen en diversas agrupaciones de intelectuales, influidos fuertemente por la discusión internacional sobre el rol del Estado (CEPAL, UNTACD; OEA), algunas organizaciones académicas (como la Universidad de Chile, en ciertas iniciativas), pero el diseño e implementación de las políticas fue siempre una tarea de los dirigentes de los partidos que accedían al poder. Los primeros intentos por evaluar los logros de las políticas públicas datan de 1990, con el establecimiento de las metas ministeriales, las que en 1995 se extendieron a los gobiernos regionales (Olavaria Gambi 2008: 3). Estas metas identificaban las prioridades de política pública que cada ministerio -y luego intendencias- debían cumplir cada año en el contexto del programa y objetivos del gobierno ${ }^{12}$.

No tenemos antecedentes que se hayan evaluado las políticas culturales como "políticas públicas", aunque se reconoce el esfuerzo que se ha hecho especialmente desde el Departamento de Estudios del CNCA por dotarse de un conjunto de herramientas valiosas para dicho cometido, como son la Cuenta Satélite de Cultura y las sucesivas encuestas de Consumo Cultural y uso del Tiempo Libre que se han realizado con el Instituto Nacional de Estadísticas.

${ }^{12}$ Para el caso chileno, las referencias más concretas sobre este tema vienen dadas por la preocupación demostrada desde 1996 en asegurar la eficacia de las leyes y la evaluación sistemática del gasto fiscal en políticas públicas. En enero y abril de 1996 la Mesa de la Cámara de Diputados y la Oficina de Informaciones organizaron un seminario sobre la eficacia de la ley que resultó señero en estas materias. Unos años más tarde, en 1998, se creó una Oficina en la Cámara dedicada el seguimiento presupuestario. 


\section{Etapa de las políticas públicas en cultura (cultural public policies)}

En estos, los primero años de la segunda década del siglo (¿tal vez una nueva etapa aún en fragua?) se asiste en Chile, no sin ciertas dificultades, a la consolidación de la institucionalidad cultural. No son del todo ajenas al discurso actual las voces de aquellos que favorecen el planteamiento de "nuevas" formas de institucionalidad de la cultura, promoviendo la creación por parte del Estado chileno, definitivamente, de la figura de un ministerio del $\mathrm{ramo}^{13}$.

Algunas naciones del subcontinente han alentado la posibilidad de emprender cambios estructurales en su institucionalidad cultural, asumiendo los llamados que ha venido haciendo la UNESCO por construir una nueva generación de políticas culturales en que el respeto a la diversidad cultural vaya junto con la diversidad ideológica ${ }^{14}$. Se trata de asumir que la promoción de la cultura también esta involucrada con la promoción de la ciencia, educación, medio ambiente, cohesión social y desarrollo sostenible.

Se asume que las nuevas políticas culturales habrán de favorecer más adecuadamente la diversidad cultural, promover un diálogo intercultural, garantizar un desarrollo sostenible y suscitar el cumplimiento de los Derechos Humanos ${ }^{15}$. Por otra parte y felizmente, en los últimos cincuenta años se ha revalorizado a la cultura como una indispensable y extraordinaria inversión para el desarrollo integral con rostro humano.

Aun más, la cultura no es considerada solamente como medio o instrumento insustituible para el desarrollo, sino como un fin último del mismo, ya que la cultura implica la significación profunda y las razones centrales de la existencia humana. En este sentido, reconocidos cientistas sociales y representantes de Organismos Internacionales vienen reiterando que la cultura representa el capital humano, el capital social y el capital creativo

${ }^{13}$ Como lo dejó entrever el propio ministro Presidente del CNCA, Luciano Cruz Coke, en la jornada inaugural de la VII Convención Nacional de la Cultura, realizada en Puerto Montt en agosto de 2010.

${ }^{14}$ El panorama cultural sufre la misma complejidad que viven los estados. Para usar la terminología constitucional, a partir de las últimas dos décadas del siglo XX y en la primera del XXI, los Estados latinoamericanos se "refundan", se "reconstituyen", se "reconocen" desde la diversidad, lo multiétnico, lo pluricultural, lo plurinacional, lo intercultural.

${ }^{15}$ Como se sabe, el decálogo de la UNESCO para el nuevo perfil de políticas culturales incluye el vínculo entre cultura y bienestar de las personas, el uso de diversos sistemas de conocimientos, interculturalidad y nuevo pensar cívico; además, crear instituciones que tomen en cuenta las relaciones interculturales, fortalecer la cultura popular y diversidad de expresiones culturales, un enfoque innovador de las políticas culturales. 
para favorecer el desarrollo económico con una gestión eficiente y eficaz, y para el logro de una convivencia democrática activa participativa y pluralista con los substantivos valores de responsabilidad social, de honestidad y transparencia y de auténtica solidaridad frente a la agobiante, dramática y generalizada crisis de valores éticos a nivel mundial y nacional.

Asistimos a lo que parece ser la conformación de una nueva generación de políticas culturales. Alejadas ya de aquellas que estuvieron orientadas a dirigir el desarrollo de las artes y la conservación del patrimonio, así como la promoción de los intercambios artísticos a comienzos del siglo XX. Fueron esas las políticas culturales del extensionismo, surgidas en la Europa de los años 50, dirigida a extender la alta cultura entre la población. De aquella época viene el gusto por los grandes equipamientos culturales, la gratuidad y las subvenciones. Centradas en la oferta cultural para hacer crecer el consumo de cultura, su principal logro fue la creación de un circuito permanente. En ellas convivió una tendencia al paternalismo de corte dirigista.

Distantes también de aquéllas formuladas en los años sesenta y setenta en Europa, las mismas que nosotros vimos un par de décadas más tarde en América Latina y que vincularon la cultura con la vida escolar, los medios y las industrias culturales. Fueron las políticas culturales del basismo.

Las políticas actuales parecen más dispuestas a asumir la complejidad de la dimensión cultural. Abiertas a reconocer las nuevas relaciones entre economía y cultura, entre cultura y trabajo, entre cultura y generación de riqueza, entre cultura y jerarquía urbana. En este nuevo cuadro que asumimos, la cultura se asume como un factor de competitividad de un territorio, como un elemento de creatividad y de innovación.

Un factor esencial en este cuadro es que las nuevas políticas públicas en cultura deberán ser evaluadas con criterios de mayor racionalidad. Si no existe un compromiso real por la evaluación de la acción cultural de los estados, mal podrán llamarse entonces "políticas públicas".

\section{CONCLUSIÓN}

Entre las experiencias que se puede sacar a propósito de lo que ha sido aquí presentado como la intervención del Estado chileno en el campo cultural, es dable reconocer que en una mirada de "larga duración" a nuestra organización política fundamental le ha interesado la cultura desde los albores de la vida institucional. Siempre ha tenido un rol de protagonista, impulsando y liderando los principales procesos vividos en la evolución de la institucio- 
nalidad chilena. Especialmente relevante ha sido la tarea conformando un marco legal, asignando recursos -ya sea directa o indirectamente- y adoptando disposiciones administrativas para la oferta de bienes y servicios culturales.

Por otra parte, esos procesos vividos en el país no son significativamente diferentes a los ocurridos en otras naciones. Ha habido un desarrollo maduro de la legislación cultural y los mecanismos de financiamiento no difieren ni en forma ni en número de los repertorios usuales que las sociedades democráticas disponen para este fin.

Pero también queda en evidencia que las políticas culturales se han hecho desde la cúspide del poder (top down), no tienen mecanismos de evaluación conocidos, no participa en ella la comunidad, no hay reportes disponibles al público y los medios de comunicación no dan mayor cobertura a estos temas. Las leyes no han resuelto los problemas de concentración de las decisiones, falta de estímulos a la demanda y ausencia de un régimen global de cooperación entre servicios públicos con competencias en el área.

Como hemos observado, la evaluación de las políticas públicas ha llegado a ser una herramienta fundamental para comprender las intervenciones del Estado en diversas áreas y sus resultados.

Si efectivamente se ha instalado ya el paradigma del diseño de las políticas públicas contemplando su evaluación y la disposición a la accountability por parte de los gobiernos, sería dable esperar a futuro que éstas sean más efectivas y eficaces.

\section{REFERENCIAS}

Abarca Besares, R. (2009, diciembre 3). Paulina Urrutia: "La cultura comienza con la democracia", Crónica. La Nación. Retrieved from http://www. lanacion.cl/noticias/site/artic/20091202/pags/20091202205948.html

Achugar, H. (2003). "Derechos culturales: ¿una nueva frontera de las políticas públicas para la cultura?". Pensar Iberoamérica 4.

Administración Pública, I. d. (2008). Glosario de términos relacionados con el seguimiento y evaluación de las políticas públicas (Glosario). Pamplona, Navarra, España: Gobierno de Navarra.

Aguilar Villanueva, L. F. (1992). La hechura de las políticas. Theodore J. Lowi. México: Miguel Angel Porrúa.

Ander-Egg, E. (2005). Política cultural a nivel municipal. Buenos Aires: Lumen. Antoine, C. (2005). Mecenazgo y patrocinio. Santiago, Chile: RIL Editores.

Arias Yurisch, K. (2010). "Revisión de las estructuras político-administrativas territoriales en el Chile del siglo XIX”. Revista Universum 25(1), 44-59. 
Arias Yurisch, K., \& Gálvez Gómez, C. (2010). Política pública cultural en Chile: Revisión de las intervenciones del Estado en el campo cultural en el siglo XX. Unpublished Postgrado, Universidad de Chile, Santiago, Chile.

Atlas, C. (2002). "Cultural Policy: What is it, Who Makes It, Why Does it matter?". Culture Counts: Strategies for a More Vibrant Cultural Life for New York City. Retrieved 6/12/2010, from http://www.nyfa.org/files_uploaded/ Pages_65-68.pdf

Baker, K. M. (1987). The French revolution and the creation of modern political culture ([1st ed.). Oxford, Inglaterra: Pergamon Press.

Ballart, X. (2005). "Evaluación de políticas. Marco conceptual y organización institucional". Revista de Estudios Políticos 80(3), 199-224.

Beaulieu, B. D. M. (2002). Histoire administrative du ministère de la culture, 1959-2002. Paris: La Documentation Francaise.

Bennett, O. (2009). "Cultural Policy in the United Kingdom: collapsing rationales and the end of a tradition". Journal of Social Policy Studies, 7(1), 65-88.

Bodo, C. (2004). politicas culturales: modelos gubernamentales. Paper presented at the Segundo Seminario de Economía de la Cultura. La tercera cara de la moneda. Retrieved from http://www.convenioandresbello.org/cab42/ index.php?option $=$ content $\&$ task $=$ view\&id $=280$

Burlot, J. G., \& Medalla, A. R. (2008). “Científicos y políticas públicas. La incidencia de una red en el tratamiento de una ley de Ordenamiento Territorial". Ordenamiento Territorial en Argentina 2(5).

Bowen Silva, M. (2008). "El proyecto sociocultural de la izquierda chilena durante la Unidad Popular: crítica, verdad e inmunología política”. Nuevo Mundo, mundos nuevos 8.

Bradford, G.; Gary, M. \& Wallach, G. (Eds.) (2000). The Politics of Culture: Policy Perspectives for Individuals, Institutions, and Communities. New York: The New York Press, XIV + 364 pp.

Burlot, J. G., \& Medalla, A. R. (2008). “Científicos y políticas públicas. La incidencia de una red en el tratamiento de una ley de Ordenamiento Territorial". Ordenamiento territorial en Argentina 2008, 2(5).

Carmona Tinoco, J. U. (2002-2003). "El derecho humano a la cultura y su protección internacional”. Revista Derecho y Cultura, Invierno.

Catalán, C., Guilisasti, R., \& Munizaga, G. (1987). Transformaciones del sistema cultural chileno entre 1920-1973. Santiago, Chile: CENECA.

Cavallo, A., Salazar, M., \& Sepúlveda, O. (2001). La historia oculta del régimen militar. Santiago: Mitos Bolsillo.

Collyer, J. (1986). Del apagón cultural a la renovación. Madrid: Centro de Estudios Salvador Allende.

David Garson, G. (1992). "De la ciencia de políticas al análisis de políticas públicas. Veinticinco años de progreso”. En Aguillar Villanueva, L. (Ed.), Antología de políticas públicas (pp. 149-180). México, DF: Miguel Angel Porrúa.

Garretón, M. A. (1992). "Estado y política cultural. Fundamentos de una nueva Institucionalidad”. Estado y política cultural en Chile. Fundamentos de una 
nueva institucionalidad (164pp.). Santiago de Chile: Ediciones División de Cultura - Ministerio de Educación.

Gelambi, M. (1999). “Politiques Públiques: Un marc conceptual”. Unpublished working paper. Departamento de Derecho Constitucional i Ciencia Politica, Universidad de Barcelona.

Gillet, J.-C., Jeannel, A., \& Ion, J. (1995). Animation et animateurs: le sens de l'action. Paris: Harmattan.

Harvey, E. (2006). "Gestión de las políticas culturales públicas en países de América y Europa. Aspectos políticos institucionales y público administrativo". Unpublished Curso de Extensión. Instituto Ortega y Gasset.

Harvey, E. (2007). "Los derechos culturales. Instrumentos normativos internacionales y políticas culturales nacionales". Paper presented at the Foro Iberoamericano de Derecho y políticas Culturales Organizado por la Secretaría de Cultura, Recreación y Deporte de Bogotá y la UCCI. Bogotá, 19 al 23 de noviembre.

Harvey, E. (2008). Instrumentos normativos internacionales y politicas culturales nacionales (Report). Ginebra, Suiza: Consejo Económico y Social, Naciones Unidas.

Henríquez Moya, R. (2003). Un estudio de caso sobre políticas culturales en Chile (1978-1990). El teatro itinerante bajo Pinochet. Santiago: Pontificia Universidad Católica de Chile.

Huerta, M. A. (2004). Las políticas públicas y las nuevas relaciones estado-sociedad: los casos de Chile, Colombia y Guatemala (1ª ed.). Bogotá: Pontificia Universidad Javeriana.

Hunter, D. (2005). “Culture war". Texas Law Review, 83(4), 1105-1136.

Ingram, D., Louis, K. S., \& Schroeder, R. G. (2004). Accountability policies and teacher decision making: Barriers to the use of data to improve practice". Teachers College Record, 106(6), 1258-1287.

Lasswell, H. D. (1971). A pre-view of policy sciences. New York; Barking: Elsevier.

Lasswell, H. D., \& Aguilar Villanueva, L. F. (1992). El estudio de las politícas públicas. México: Miguel Ángel Porrúa.

Lindblom, C. (1996). "La ciencia de 'salir del paso'”. In Aguilar Villanueva, L. F. (Ed.), El estudio de las políticas públicas. México DF: Porrúa.

Mark, C. C. (1969). A study of cultural policy in the United States. París, Francia: UNESCO.

Negrier, E. (2003). "Las políticas culturales en Francia y España: una aproximación nacional y local comparada". Barcelona: Institut de Ciencies Politiques i Socials (ICPS).

Ochoa Gautier, A. M. (2003). Entre los deseos y los derechos. Un ensayo crítico sobre las políticas culturales. Bogotá, Colombia: Instituto Colombiano de Antropología e Historia.

Olavarría Gambi, M. (2008). "La evaluación de políticas públicas en Chile". Paper presented at the XIII Congreso Internacional del CLAD sobre la Reforma del Estado y de la Administración pública. 
Polle, F., \& Fernández Jilberto, A. (1992). “Transición a la democracia en una economía neoliberal: el caso de Chile”. Sistema: Revista de Ciencias Sociales (108), 85-106.

Prieto de Pedro, J. (2009). "El derecho de la cultura como nueva especialidad jurídica”. In Asuaga, C. (Ed.), Un encuentro no casual. Cultura, ciencias económicas y derecho. Actas Primer Seminario Ciencias Económicas, Derecho y Cultura: Hacia un modelo del Río de la Plata (pp. 134). Montevideo: Universidad de la República.

Quade, E. (1989). Análisis de formación de decisiones politicas. Madrid: Instituto de Estudios Fiscales.

Rausell Koster, P. (2004). El envés de la cultura (10 años de economía de la cultura en Valencia). Valencia: Ediciones Mínim.

Rubinich, L. (1993). Extensionismo y basismo: dos estilos de politica cultural. Buenos Aires: Editorial Espacio.

Solarte Pazos, L. (2004). Las evaluaciones de políticas públicas en el Estado liberal. Bogotá, Colombia.

Squella, A. (2005). El jinete bajo la lluvia. La cultura en el gobierno de Ricardo Lagos. Santiago, Chile: Aguilar. . (2006). "La nueva institucionalidad cultural". En VVAA (Ed.). La cultura durante el período de la transición a la democracia 1990-2005 (pp. 407). Santiago de Chile: Consejo Nacional de la Cultura y las Artes.

Stanziola, J. (2002). "Neo-liberalism and cultural policies in latin america: The case of Chile". International Journal of Cultural Policy, 8(1), 21-35.

Subercaseaux, B. (1991). Política y Cultura. Desencuentros y aproximaciones. Revista Nueva Sociedad(116), 144-145.

Subercaseaux, B. (1993). Historia del libro en Chile (alma y cuerpo). Santiago de Chile: Editorial Andrés Bello.

Subercaseaux, B. (1997). Historia de las ideas y de la cultura en Chile Santiago de Chile: Editorial Universitaria.

Subercaseaux, B. (2006). "La cultura en los gobiernos de la Concertación”. Universum. Revista de Humanidades y ciencias sociales 1, 191-203.

UNESCO (1982). Declaración de México sobre las Políticas Culturales. México D.F., 6 de agosto.

Urteaga, U. (2009). "La evaluación de las políticas públicas: principios metodológicos”. SEE-Valuación. Revista de la Sociedad Española de Evaluación 9.

Vedung, E. (1986). "Development of the methods of evaluation" (Meléndez, C., Trans.). In O. E. a. C. A. Swedish Ministry (Ed.), Methods for Evaluation of National Cultural Policies (pp. 95-97). Stockholm, Sweden: Swedish Ministry, Of Education and Cultural Affairs.

Yoshimoto, M. (2005). "The Evaluation of Cultural Institutions and PoliciesToward a Framework for Creative Evaluation”. Social Development Research Department, Reino Unido.

Yúdice, G., \& Miller, T. (2004). Política cultural. Barcelona: Gedisa. 